\title{
Orientation-Selective Adaptation to Illusory Contours in Human Visual Cortex
}

\author{
Leila Montaser-Kouhsari, ${ }^{1}$ Michael S. Landy, ${ }^{1,2}$ David J. Heeger, ${ }^{1,2}$ and Jonas Larsson ${ }^{1,2}$ \\ ${ }^{1}$ Department of Psychology and ${ }^{2}$ Center for Neural Science, New York University, New York, New York 10003
}

\begin{abstract}
Humans can perceive illusory or subjective contours in the absence of any real physical boundaries. We used an adaptation protocol to look for orientation-selective neural responses to illusory contours defined by phase-shifted abutting line gratings in the human visual cortex. We measured functional magnetic resonance imaging (fMRI) responses to illusory-contour test stimuli after adapting to an illusory-contour adapter stimulus that was oriented parallel or orthogonal to the test stimulus. We found orientation-selective adaptation to illusory contours in early (V1 and V2) and higher-tier visual areas (V3, hV4, V01, V3A/B, V7, L01, and L02). That is, fMRI responses were smaller for test stimuli parallel to the adapter than for test stimuli orthogonal to the adapter. In two control experiments using spatially jittered and phase-randomized stimuli, we demonstrated that this adaptation was not just in response to differences in the distribution of spectral power in the stimuli. Orientation-selective adaptation to illusory contours increased from early to higher-tier visual areas. Thus, both early and higher-tier visual areas contain neurons selective for the orientation of this type of illusory contour.
\end{abstract}

Key words: illusory contours; adaptation; orientation selectivity; fMRI; visual cortex; spatial vision

\section{Introduction}

Illusory contours are perceived as sharp boundaries between two regions that do not differ in mean luminance or chromaticity. For example, two phase-shifted abutting line gratings (Fig. $1 \mathrm{~A}$ ) elicit a clear boundary percept in the absence of any average luminance difference across the boundary (Kanizsa, 1976; Soriano et al., 1996). In this paper, we report that regions of human visual cortex respond selectively to the orientation of such illusory contours.

Single-unit recordings have shown that V2 neurons selectively respond to the orientation of both real and illusory contours (von der Heydt and Peterhans, 1989; Lee and Nguyen, 2001). The existence of such "illusory-contour" cells in V1 is more controversial. Some authors claim that illusory-contour cells are absent in V1 (Peterhans and von der Heydt, 1989; von der Heydt and Peterhans, 1989), but others report that some V1 cells respond to illusory contours (Grosof et al., 1993; Sheth et al., 1996; Lee and Nguyen, 2001; Ramsden et al., 2001). Single-unit responses to illusory contours have not been measured beyond V2.

Several imaging studies of illusory contours have been done in humans (Hirsch et al., 1995; Ffytche and Zeki, 1996; Larsson et al., 1999; Mendola et al., 1999; Seghier et al., 2000; Stanley and Rubin, 2003; Maertens and Pollmann, 2005). As a whole, these studies reported responses to illusory contours in V1 and V2 as well as higher-tier visual areas, including the lateral occipital

Received Sept. 22, 2006; revised Jan. 16, 2007; accepted Jan. 17, 2007.

This work was supported by National Institutes of Health Grant EY16165 (M.S.L., D.J.H.) and by the Seaver Foundation. We thank Justin Gardner and Tony Movshon for their helpful comments.

Correspondence should be addressed to Leila Montaser-Kouhsari, Department of Psychology, New York University, 6 Washington Place, Room 957, New York, NY 10003. E-mail: Imk306@nyu.edu. DOI:10.1523/JNEUROSCI.4173-06.2007

Copyright $\odot 2007$ Society for Neuroscience $\quad 0270-6474 / 07 / 272186-10 \$ 15.00 / 0$ complex (LOC), but responses were stronger in higher-tier areas (e.g., V3A, LOC) than in early visual areas (V1 and V2).

It is difficult to compare the results of the neuroimaging and single-unit studies. Previous neurophysiological studies reported orientation-selective responses of neurons to illusory contours. In contrast, imaging studies compared brain activity in response to illusory-contour stimuli versus control stimuli that did not evoke illusory contour percepts. However, an overall net increase in neural activity within a cortical area to illusory contour stimuli may or may not be indicative of contour processing per se. First, any of a number of differences between the illusory-contour and control stimuli (e.g., differences in the orientation and spatial frequency content of the stimuli) might affect the overall level of activity and thereby confound the interpretation of the results. Second, illusory contours are compelling visual illusions such that attentional modulation of neural activity might confound the interpretation (Huk et al., 2001). Third, most models of visual processing hypothesize that a contour percept is encoded by a relative difference between subpopulations of neurons with different orientation preferences, not by an overall net increase in activity (Gilbert and Wiesel, 1990; Vogels, 1990; Pouget et al., 2000).

We used adaptation to measure orientation-selective responses to illusory contours in human visual cortex while observers performed a demanding foveal task to equate spatial attention across conditions (Larsson et al., 2006). We found orientationselective adaptation to illusory contours in early (V1 and V2) and higher-tier (V3, V4, VO1, V3A/B, V7, LO1, and LO2) visual areas. Orientation-selective adaptation increased from early to higher-tier visual areas, implying that the proportion of neurons selective for illusory-contour orientation was larger in higher-tier visual areas. Our results are consistent with single-cell studies that have shown orientation-selective responses to illusory contours 
A

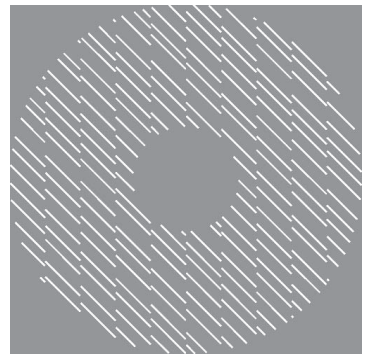

B

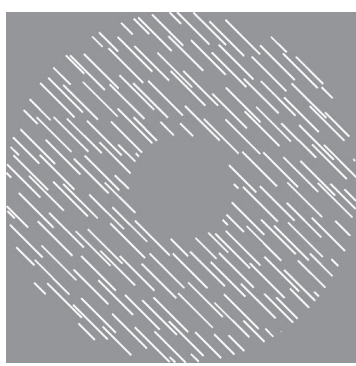

C

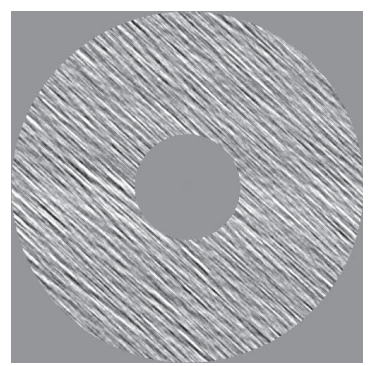

Figure 1. A, Example illusory-contour stimulus. Abutting line gratings elicited perception of illusory contours (in this case vertical). $\boldsymbol{B}$, Example adapter stimulus used in the first control experiment that did not evoke illusory-contour percepts. These stimuli were made by misaligning the inducers, shifting each inducer line parallel to its orientation across the illusory boundaries. C, Example adapter stimulus used in the second control experiment. These stimuli were random-noise patterns with the same power spectrum as the illusory-contour stimuli but with randomized phase spectrum.

A

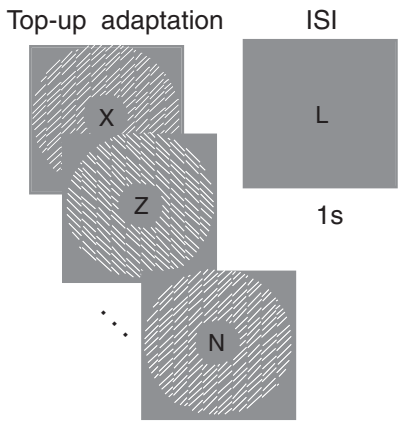

$4 \mathrm{~s}$

B

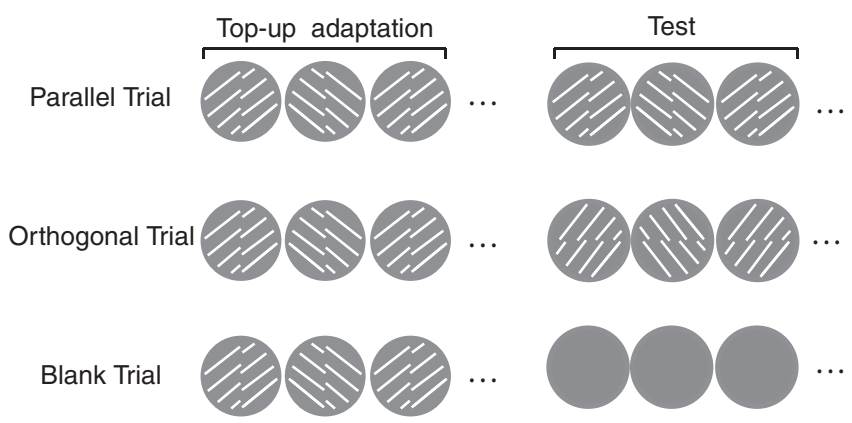

Figure 2. Experimental protocol. $\boldsymbol{A}$, Each trial consisted of top-up adaptation followed by a test stimulus. To control and equate attention across trials, observers performed an attention-demanding task at fixation, counting the number of $X$ s shown in a stream of rapidly presented letters $(Z, L, N, T$, and $X)$, each presented for $160 \mathrm{~ms}$. The letters were shown throughout each trial, from the beginning of the adapter until the end of presentation of the test stimulus (see Materials and Methods). ISI, Interstimulus interval. $B$, Schematics of the three trial types. To avoid adaptation to the inducers, the orientation of the inducer lines changed every 160 ms during the adaptation and test period.

in V1 and V2 and also confirm imaging studies that have shown activation of higher-tier visual areas in response to illusory contours.

\section{Materials and Methods}

Observers and scanning sessions. Four observers (two male and two female, 27-49 years of age) participated in the main experiment and the first control experiment. Two of the observers (one male and one female) also participated in the second control experiment. Each experiment (main and two control experiments) consisted of two scanning sessions. Observers gave informed consent to participate in accordance with the Helsinki convention and National Institutes of Health guidelines for experiments involving human subjects. The experimental protocol was $157.5 \mathrm{~cm}$ approved by the New York University Committee on Activities Involving Human Subjects.

Visual stimuli. Illusory-contour stimuli were presented in an annulus (inner radius, $1.5^{\circ}$; outer radius, $4.9^{\circ}$ ) around the center of fixation (Fig. 1A). Stimuli were arrays of inducer lines, white diagonal $\left(+45\right.$ or $\left.-45^{\circ}\right)$ line segments ( 0.23 arc min wide; $1.4^{\circ}$ long, $50 \%$ contrast) on a uniform gray background, arranged to form horizontal or vertical abutting line gratings phase shifted by $180^{\circ}$, resulting in the percept of crisp illusory contours between adjacent inducer gratings. The position of each line was jittered by a random amount between $\pm 0.15^{\circ}$, parallel to the orientation of the induced illusory contours. This jitter obscured the regularity of the inducer grating pattern, which might have provided a cue to the orientation of the illusory contours even in the absence of an illusory-contour percept. The spatial frequency of the inducer gratings was $2.8 \mathrm{cycles} /{ }^{\circ}$ on average (ignoring the jitter). The spatial frequency of illusory contours was 1 contour ${ }^{\circ}$.

In addition to the main experiment, we ran two control experiments that used adapter stimuli [see functional magnetic resonance imaging (fMRI) protocol below] designed to be similar to those in the main experiment but did not induce the perception of illusory contours. The test stimuli in these control experiments were the same illusory-contour stimuli that were used in the main experiment; only the adapter stimuli differed. In the first control experiment, each inducer line was shifted (misaligned), parallel to its orientation, by a random amount ranging from -0.55 to $0.55^{\circ}$ (Fig. $1 B)$. By misaligning the endpoints of inducer lines, this manipulation eliminated the percept of illusory boundaries between adjacent inducer gratings. As in the main experiment, the orientation of the inducers was either +45 or $-45^{\circ}$. In the second control experiment, the adapter stimuli were random-noise patterns generated by randomizing the phase spectrum of the corresponding illusory-contour stimuli (Fig. 1C).

For a separate psychophysical experiment, stimuli were generated with seven levels of misalignment, ranging from no misalignment, as in the main experiment (Fig. $1 \mathrm{~A}$ ), to the misalignment that was used for the first control experiment (Fig. $1 B$ ). As in the first control experiment, each inducer line was shifted parallel to its orientation by a random amount drawn from a uniform distribution.

For the fMRI experiments, stimuli were presented on an electromagnetically shielded analog NEC 2110 LCD display using the Psychophysics Toolbox (Brainard, 1997; Pelli, 1997) and a 10-bit graphics card. For the psychophysical experiments, stimuli were presented on a Nokia (Finland) 446 XPro CRT display. Both displays had a refresh rate of $60 \mathrm{~Hz}$ and spatial resolution of $800 \times 600$ pixel. The mean luminance of the display background was $18.14 \mathrm{~cd} / \mathrm{m}^{2}$ in the fMRI experiments and $51.81 \mathrm{~cd} / \mathrm{m}^{2}$ in the psychophysical experiments. The viewing distance for both the fMRI and psychophysical experiments was

fMRI protocol. We used an event-related fMRI adaptation protocol (Fig. 2) (Engel, 2005; Fang et al., 2005; Gardner et al., 2005; Larsson et al., 2006). In this protocol, observers viewed one illusory-contour stimulus repeatedly (the adapter), and we measured the fMRI response to a sub- 


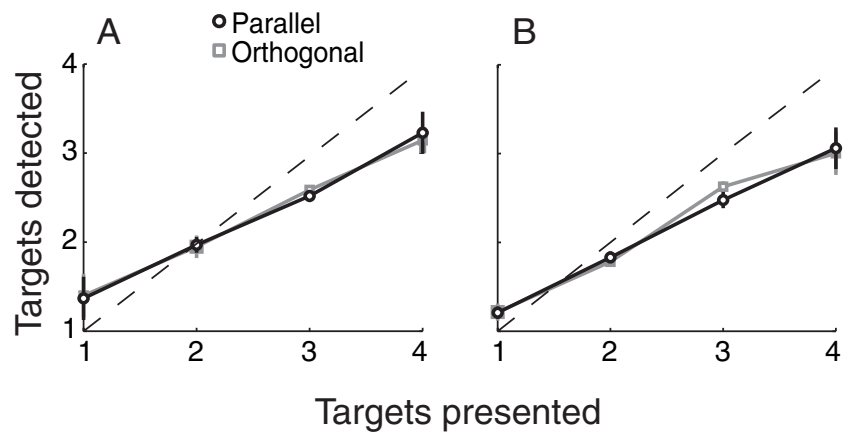

Figure 3. Task performance. The number of targets detected is plotted as a function of number of targets displayed for both the parallel (black) and orthogonal (gray) trials. The dashed line indicates perfect performance. Error bars represent SEM across four observers. $A$, Main experiment. $\boldsymbol{B}$, First control experiment.

sequently presented illusory-contour stimulus (the test) that had either the same ("parallel") or perpendicular ("orthogonal") illusory-contour orientation as the adapter.

At the beginning of each session, observers passively viewed the adapter stimulus for $100 \mathrm{~s}$. Adaptation was maintained on subsequent trials by showing a top-up adapter during the first $4 \mathrm{~s}$ of each trial. A blank (uniform gray) screen followed the top-up adapter for $1 \mathrm{~s}$, which was followed by the test stimulus for $1 \mathrm{~s}$. The trial ended with a $1.2 \mathrm{~s}$ behavioral-response period, during which the display was blank except for the fixation point (Fig. 2A). Total trial duration was $7.2 \mathrm{~s}$. To avoid adaptation to the inducers (independent of adaptation to the illusory contours), the orientation of the inducers was changed every $160 \mathrm{~ms}$ during both the adapter and test stimuli (Fig. 2A) while keeping the illusory-contour orientation constant. In addition, the spatial phase of the illusory contours and that of the inducers was varied randomly every $160 \mathrm{~ms}$.

On one-third of the trials, the test stimulus had the same orientation as the adapter (parallel trials); on one-third, the test stimulus was perpendicular to the adapter (orthogonal trials); and on one-third, the test stimulus was blank (adapter-only trials) (Fig. 2B). The trial order was randomly shuffled so that the sequence of trials preceding and following any trial was equally likely to contain any of the three trial types. Specifically, 14 trials of each trial type were presented in seven blocks of six trials each, with trial order randomized within blocks.

Each observer participated in two scanning sessions, one with a horizontal adapter and the other with a vertical adapter. A single adapter orientation, vertical or horizontal, was used for each scanning session. A scanning session comprised 10 adaptation scans (runs), each consisting of 42 trials $(\sim 5 \mathrm{~min}$ ), plus two localizer scans (see below). The data were averaged across the two sessions for each observer (i.e., across 840 trials).

To control attention, observers performed an attention-demanding task at fixation (Fig. $2 \mathrm{~A}$ ) that forced them to divert their attention away from the peripheral illusory-contour stimuli. This was necessary, because spatial attention can modulate neuronal responses to visual stimuli measured with fMRI in a spatially specific manner (Tootell et al., 1998; Brefczynski and DeYoe, 1999; Gandhi et al., 1999; Kastner et al., 1999; Somers et al., 1999). Furthermore, attention modulates aftereffects, including the motion aftereffect (Chaudhuri, 1990; Lankheet and Verstraten, 1995; Rees et al., 1997), figural aftereffect (Shulman, 1992; Yeh et al., 1996; Suzuki, 2001), tilt aftereffect (Spivey and Spirn, 2000), and illusorycontour tilt aftereffect (Montaser-Kouhsari and Rajimehr, 2004). In our task, observers were required to count the number of Xs in a stream of rapidly (160 ms/letter) presented letters $(\mathrm{Z}, \mathrm{L}, \mathrm{N}, \mathrm{T}$, and $\mathrm{X})$. The letters were shown from the beginning of the adapter until the end of the test stimulus. Afterward, the letters were replaced by a fixation point, cueing observers to respond by pressing one of four keys corresponding to the number of target Xs detected (1-4). Despite the great attentional and working-memory demands of the task, observers' performance after practice was well above chance (Fig. 3). Importantly, there was no difference in performance between the two experimental conditions, indicat- ing that there was no difference in attentional resources devoted to the task between the parallel and orthogonal conditions that could contribute to differences in fMRI responses that we measured. This also indicates that there was no difference in eye position between the conditions, because breaking fixation would have adversely affected performance. Hence, eye movements did not confound the interpretation of our fMRI results.

We ran two control experiments in which the trial sequence, task, and experimental protocol were identical to the main experiment. In the first control experiment, we used the misaligned stimuli (Fig. $1 B$ ) as adapters and the same illusory-contour test stimuli as before. These adapter stimuli were visually similar to the illusory-contour adapter stimuli except for the lack of illusory contours. In the second control experiment, the adapter stimuli had identical power spectra as the illusory-contour stimuli, but the phase spectra were randomized (Fig. 1C). The idea behind both manipulations was to ensure that any adaptation effects observed in the main experiment reflected orientation-selective responses to the perceived illusory contours, rather than being a result of incidental features or first-order (luminance contrast) artifacts in the stimuli. As in the main experiment (see above), each observer participated in two scanning sessions for each control experiment, one with a horizontal adapter and the other with a vertical adapter. Each scanning session comprised 10 scans with 42 trials per scan, as in the main experiment.

Localizer scans. During each scanning session, before and after the adaptation scans, we performed an additional pair of measurements to independently identify the cortical regions responding to the stimuli. The same abutting line-grating illusory-contour stimuli were used as in the main experiment. A block design was used, alternating between $9.6 \mathrm{~s}$ blocks of illusory-contour stimuli and $9.6 \mathrm{~s}$ blocks of blank (uniform gray) screen. Observers were instructed to maintain their gaze on a fixation mark at the center of the display throughout the scan. Each localizer scan consisted of 13 stimulus-blank alternations.

MRI acquisition. A Siemens (Erlangen, Germany) Allegra 3T scanner was used to measure the blood oxygenation level-dependent (BOLD) signal (Ogawa et al., 1990) in $\mathrm{T} 22^{*}$-weighted images. The scanner was equipped with a four-channel phased-array surface coil covering the back of the head (NM-011 transmit head coil and NMSC-021 receive coil; Nova Medical, Wakefield, MA). A bite bar was used to minimize head motion. Functional data in the adaptation scanning sessions were acquired using the following parameters: repetition time (TR), $1200 \mathrm{~ms}$; echo time (TE), $30 \mathrm{~ms}$; flip angle, $75^{\circ}$; $64 \times 64$ matrix size; 19 slices oriented perpendicular to the calcarine sulcus; voxel size, $3 \times 3 \times 3 \mathrm{~mm}$. For the retinotopy measurements (see below), we used the same imaging parameters with the following exceptions: 24 slices and $1500 \mathrm{~ms}$ TR. At the beginning of each session, we also acquired anatomical T1-weighted magnetization-prepared rapid-acquisition gradient echo images that covered the same volume as the functional scans but with twice the in-plane resolution (voxel size, $1.5 \times 1.5 \times 3 \mathrm{~mm}$ ).

Defining visual area regions of interest. Nine regions of interest (ROIs) were defined based on retinotopy (V1, V2, V3, V3A/V3B, hV4, V7, LO1, LO2, and VO1). Standard traveling wave methods for retinotopic mapping were used to identify meridian representations corresponding to boundaries between retinotopically organized visual areas (Engel et al., 1994, 1997; Sereno et al., 1995; DeYoe et al., 1996). The retinotopic mapping scans were obtained in scanning sessions separate from the adaptation sessions. The ROIs were drawn on computationally flattened representations ("flat maps") of the occipital cortex generated from high-resolution T1-weighted anatomy images using the public domain software SurfRelax (Larsson, 2001) (http://www.cma.mgh.harvard.edu/ iatr). Areas V1, V2, V3, V3A/V3B, hV4, and V7 have been described extensively in the literature (Wandell et al., 2005). VO1 is a coarsely retinotopic area anterior and lateral to hV4 (Wandell et al., 2005). LO1 and $\mathrm{LO} 2$ are two retinotopic areas in the lateral occipital cortex between dorsal V3 and V5/MT+ (Larsson and Heeger, 2006; Larsson et al., 2006).

fMRI data analysis. The time-series data from each scan were motion corrected within and between scans (Jenkinson et al., 2002). The time series of each voxel was normalized (divided by its mean intensity) to compensate for variations in intensity with distance from the receiver 
coil and to convert the data from arbitrary image intensity units to percentage signal change.

For the retinotopy and localizer scans, the time-series data from each voxel were highpass filtered (cutoffs at 0.026 and $0.02 \mathrm{~Hz}$ for the retinotopy and localizer scans, respectively) to remove low-frequency noise and drift (Zarahn et al., 1997; Smith et al., 1999) and then were fit with a sinusoid with period equal to that of the stimulus (Bandettini et al., 1993; Engel et al., 1994) yielding both the phase of the best-fit sinusoid and the correlation (technically, coherence) between the best-fit sinusoid and the measured time series. The coherence reflected the signal-to-noise ratio of the evoked responses (Engel et al., 1997), ranging from 0 (no modulation) to 1 (perfect coherence). The best-fit phase measured the temporal delay of the fMRI signal relative to the stimulus cycle. For the retinotopy scans, the phase corresponded to the polar angle and radial components of the retinotopic map. For the localizer experiments, response phase reflected the stimulus condition that evoked the larger response (illusory-contour stimuli or blank field).

Event-related data from the adaptation scans were analyzed separately for each visual area ROI as follows. For each adaptation session, the ROI was restricted to include only those voxels that responded strongly in the localizer scans taken in the same session. Specifically, the ROIs were restricted to include voxels with a response coherence $>0.2$ and phase between 0 and $\pi$, corresponding to stimulus "on" blocks. This ensured that ROIs included only voxels corresponding to visual field locations within the stimulus annulus and reduced intersession variability (Aguirre et al., 1998) by excluding voxels that did not respond strongly to the stimuli. The normalized time courses were averaged across voxels within each ROI. The resulting mean ROI time courses were filtered using a bandpass filter $(0.02-0.2 \mathrm{~Hz})$ to remove low-frequency drift and high-frequency noise. Responses to individual trials were extracted from the mean ROI time course by extracting the 16 time points $(19.2 \mathrm{~s}$ ) beginning with the onset of each trial. For each observer, parallel, orthogonal, and blank trials were each pooled across the two scanning sessions (vertical and horizontal adapters). We estimated the response to the adapter stimulus as the mean response for the adapter-only (blank) trials. The mean response to the adapter stimulus was subtracted from the response to each orthogonal and parallel trial, and the resulting time courses were adjusted to a zero baseline by subtracting the mean of the first four time points (corresponding to the time of presentation of the $4 \mathrm{~s}$ adapter and the $1 \mathrm{~s}$ blank between adapter and test). The peak response amplitudes evoked by the test stimuli (orthogonal or parallel) for each trial were then computed by averaging the two values of the time course around the peak. These two time points, which were the same for all of the ROIs and observers, corresponded to 4.6 and $5.8 \mathrm{~s}$ after test stimulus onset (i.e., typical of the peak hemodynamic impulse response). The response amplitudes of individual parallel and orthogonal trials were averaged by trial type to compute the mean response amplitude for each trial type for each visual area ROI. Confidence intervals for mean response amplitudes were calculated for individual observers as the SEM across trials. We estimated the statistical significance of adaptation (i.e., larger response amplitudes to orthogonal than to parallel trials) using a one-tailed $t$ test in individual observers. For group means, we averaged the response amplitudes across the four observers. Confidence intervals for the group amplitude means were computed as the confidence interval pooled across observers (i.e., by taking the square root of the mean squared SEMs of individual observers divided by square root of the number of observers).

We also computed an adaptation index (AI) for each visual area to quantify the difference between the fMRI responses to parallel and orthogonal test stimuli as a result of adaptation in each visual area. The
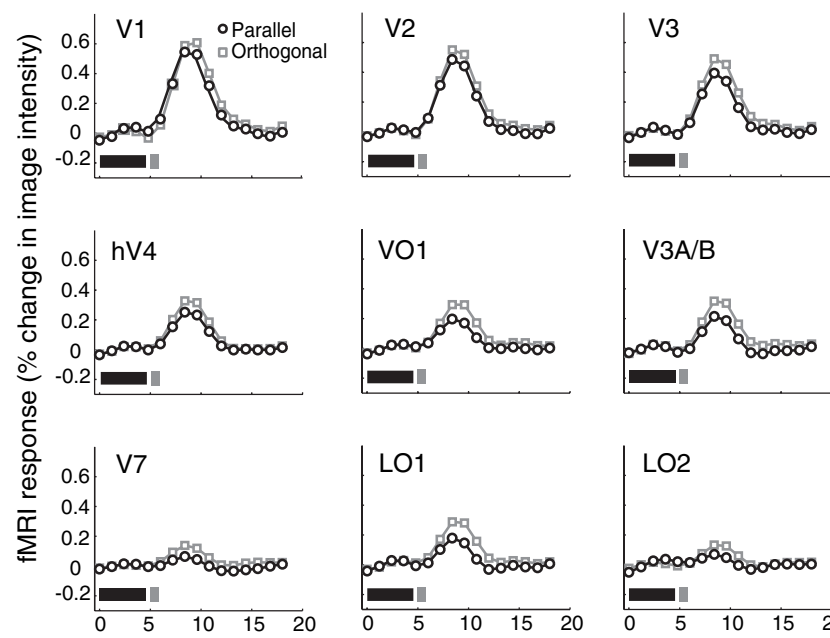

LO1

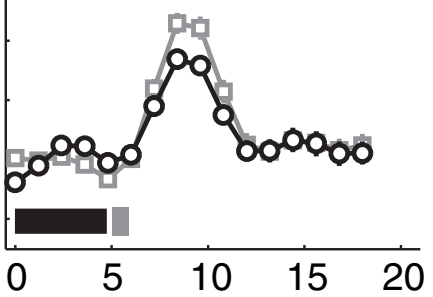

Time (s)

\section{0}
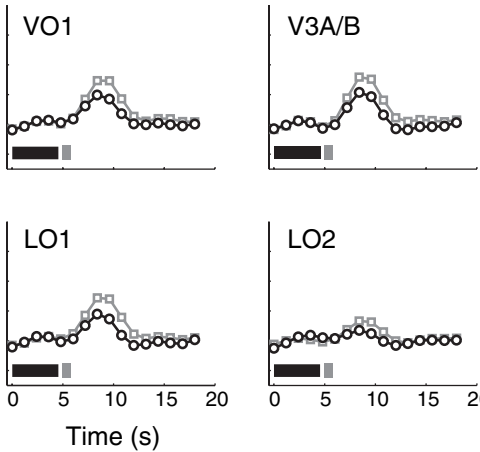

LO2

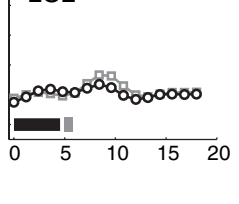

Figure 5. Time courses of fMRI responses, averaged across observers, from the main experiment for visual areas V1, V2, V3, hV4, V01, V3A/B, V7, L01, and L02. Same conventions are used as in Figure 3, except error bars reflect SEM across four observers. Measured responses to orthogonal test stimuli were significantly larger than responses to parallel test stimuli in all of the retinotopic visual areas.

index was calculated as follows: $\mathrm{AI}=\left(A_{O}-A_{P}\right) /\left(\left|A_{O}\right|+\left|A_{P}\right|\right)$, where $A_{P}$ was the mean response amplitude to the parallel stimulus, and $A_{O}$ was the mean response amplitude to the orthogonal stimulus. This index ranged from -1 to 1 . The index was one if adaptation was completely effective, reducing the response to the parallel stimulus to zero. If adaptation was ineffective, so that responses to the parallel and orthogonal stimuli were identical, the index was zero.

For each ROI, the adaptation indices were computed separately for each observer and then averaged. Confidence intervals for adaptation indices were estimated using a bootstrap method (Efron and Tibshirani, 1993). We estimated the underlying distribution of the adaptation indices by resampling the data 1000 times. The response amplitudes from each ROI and each observer were sampled with replacement to yield a resampled data set with the same size as the original data. The resampled adaptation indices were averaged across the four observers, yielding a distribution of 1000 adaptation indices for each ROI. Upper and lower 
Table 1. Response-amplitude difference (percentage change in image intensity) between orthogonal and parallel trials, in main and both control experiments, for individual observers and averaged across observers for all visual areas

\begin{tabular}{|c|c|c|c|c|c|c|c|c|c|}
\hline & V1 & V2 & V3 & V4 & V01 & V3AB & V7 & L01 & L02 \\
\hline \multicolumn{10}{|c|}{ Main experiment } \\
\hline Observer 1 & $0.08(0.155)$ & $0.08(0.044)$ & $0.12(0.004)$ & $0.06(0.039)$ & $0.07(0.042)$ & $0.1(0.035)$ & $0.08(0.050)$ & $0.12(0.006)$ & $0.07(0.108)$ \\
\hline Observer 2 & $0.09(0.067)$ & $0.1(0.017)$ & $0.1(0.009)$ & $0.11(0.000)$ & $0.14(0.001)$ & $0.05(0.158)$ & $0.02(0.354)$ & $0.08(0.048)$ & $0.02(0.402)$ \\
\hline Observer 3 & $0.08(0.089)$ & $0.1(0.038)$ & $0.15(0.009)$ & $0.12(0.004)$ & $0.13(0.002)$ & $0.2(0.000)$ & $0.14(0.003)$ & $0.18(0.007)$ & $0.14(0.003)$ \\
\hline Observer 4 & $-0.01(0.564)$ & $0.01(0.430)$ & $0.05(0.059)$ & $0.03(0.305)$ & $0.1(0.020)$ & $0.09(0.020)$ & $0.08(0.034)$ & $0.1(0.002)$ & $0.07(0.051)$ \\
\hline Group & $0.06(0.023)$ & $0.07(0.001)$ & $0.1(0.000)$ & $0.08(0.000)$ & $0.11(0.000)$ & $0.11(0.000)$ & $0.08(0.000)$ & $0.12(0.000)$ & $0.07(0.006)$ \\
\hline \multicolumn{10}{|c|}{ First control experiment } \\
\hline Observer 1 & $-0.06(0.775)$ & $0.02(0.360)$ & $-0.02(0.624)$ & $-0.01(0.604)$ & $-0.06(0.965)$ & $-0.07(0.908)$ & $-0.07(0.974)$ & $-0.05(0.894)$ & $0.08(0.959)$ \\
\hline Observer 2 & $0.06(0.173)$ & $0.04(0.267)$ & $0.05(0.209)$ & $0.03(0.205)$ & $0.07(0.085)$ & $0.01(0.434)$ & $0.08(0.067)$ & $0.00(0.486)$ & $0.02(0.324)$ \\
\hline Observer 3 & $0.00(0.504)$ & $-0.02(0.625)$ & $-0.06(0.828)$ & $-0.04(0.803)$ & $-0.04(0.775)$ & $-0.04(0.742)$ & $-0.05(0.827)$ & $-0.07(0.824)$ & $-0.03(0.741)$ \\
\hline Observer 4 & $0.05(0.211)$ & $0.09(0.087)$ & $0.08(0.070)$ & $0.06(0.168)$ & $0.04(0.277)$ & $0.08(0.126)$ & $0.08(0.116)$ & $0.09(0.063)$ & $0.14(0.042)$ \\
\hline Group & $0.02(0.309)$ & $0.03(0.122)$ & $0.01(0.316)$ & $0.01(0.314)$ & $0.00(0.430)$ & $-0.00(0.537)$ & $0.01(0.338)$ & $-0.01(0.566)$ & $0.01(0.306)$ \\
\hline \multicolumn{10}{|c|}{ Second control experiment } \\
\hline Observer 2 & $-0.1(0.890)$ & $-0.07(0.834)$ & $-0.03(0.684)$ & $0.02(0.359)$ & $0.01(0.410)$ & $-0.02(0.626)$ & $-0.01(0.558)$ & $0.00(0.511)$ & $-0.3(0.684)$ \\
\hline Observer 4 & $0.09(0.166)$ & $0.05(0.286)$ & $0.04(0.310)$ & $0.03(0.400)$ & $0.01(0.451)$ & $0.04(0.348)$ & $0.07(0.226)$ & $0.05(0.293)$ & $0.09(0.153)$ \\
\hline Group & $0.00(0.503)$ & $-0.01(0.556)$ & $0.01(0.453)$ & $0.02(0.353)$ & $0.01(0.416)$ & $0.01(0.441)$ & $0.03(0.309)$ & $0.02(0.331)$ & $0.03(0.259)$ \\
\hline
\end{tabular}

$p$ values (numbers in parentheses) indicate probability of obtaining a smaller response to parallel trials than to orthogonal trials by chance (for individual observers and averaged group): one-tailed $t$ test; bold type indicates a significantly $(p<0.05)$ larger response to orthogonal than to parallel trials.

error bounds for the mean adaptation indices were estimated as the 16 th and 84 th percentiles of the resulting distributions.

Psychophysical protocol. To verify that the adapter stimuli were behaviorally effective in eliciting adaptation to illusory contours in the main experiment but not in the first control experiment, we measured postadaptation discrimination thresholds to the stimuli with various amounts of misalignment. The stimuli and protocol were similar to those in the fMRI experiments.

Observers performed a two-interval forced-choice task. Two stimuli were displayed in succession. One had the maximum amount of misalignment $\left(0.55^{\circ}\right)$ and the other (the target) had a smaller amount of misalignment. Observers indicated which stimulus was less misaligned (i.e., the stimulus that had the stronger percept of illusory contours). Before each session, observers viewed an adapter stimulus for $100 \mathrm{~s}$. The adapters were either the illusory contours used in the main fMRI experiment or the misaligned adapter stimuli used in the first control experiment. Each trial was $6.6 \mathrm{~s}$ long and began by presenting a top-up adapter for $4 \mathrm{~s}$, followed by a blank screen for $0.5 \mathrm{~s}$, followed by two test stimuli for $0.5 \mathrm{~s}$ each, separated by an interstimulus interval of $0.5 \mathrm{~s}$. At the end of each trial, observers indicated by key press which interval contained the (less misaligned) illusory-contour target. The misalignment in the target stimulus was varied by two interleaved one-up, two-down staircases (Wetherill and Levitt, 1965). A single experimental session consisted of 10 blocks, each with 20 trials. Target orientation alternated from block to block but was constant within a block. Each observer participated in at least four experimental sessions (one for each combination of adapter type and adapter orientation). The sessions were run on different days to avoid potential confounding effects of long-term adaptation. There were at least 100 trials for each combination of adapter type (illusory contour or misaligned), adapter orientation (horizontal or vertical), and teststimulus orientation (orthogonal or parallel). Results were pooled across adapter orientations, and psychometric functions were fit to the data using a maximum-likelihood method (Wichmann and Hill, 2001a,b) (http://bootstrap-software.org/psignifit). Discrimination thresholds were defined as the amount of misalignment corresponding to $75 \%$ correct responses.

\section{Results}

\section{Cortical activations evoked by localizer stimuli}

To identify the cortical regions responding to the illusorycontour stimulus, we measured responses evoked by a localizer stimulus (illusory contours alternating with a blank, uniform gray screen; see Materials and Methods). Localizer stimuli evoked robust contiguous activity across retinotopic visual areas. The spatial pattern of the evoked responses was similar in different scanning sessions and across observers. In early visual areas, we found a band of decreased activity peripheral and foveal to the region of increased activity. This decrement in activity may be attributable to hemodynamic effects (e.g., "blood stealing") or decreases in neuronal activity (Shmuel et al., 2002, 2006). We defined ROIs based on the localizer responses in each visual cortical area (see Materials and Methods).

\section{Orientation-selective fMRI response adaptation to illusory contours}

We found evidence of orientation-selective adaptation to illusory contours in multiple visual areas. Time courses from V1 and LO1 for one observer are shown in Figure 4. Note that the fMRI responses are larger for the orthogonal (unadapted) than the parallel (adapted) stimuli, a signature of orientation-selective adaptation. The differences between the responses to parallel versus orthogonal test stimuli were not significant in individual observers in visual area V1 but were statistically significant when averaged across observers. In visual area LO1, the response differences were significant both in the pooled data and in all four observers.

When pooled across observers, the fMRI responses showed significant adaptation in all visual cortical areas studied (Fig. 5, Table 1). The group-averaged time courses for three early visual areas (V1, V2, and V3), two ventral higher-tier areas (hV4 and $\mathrm{VO} 1)$, and two dorsal (V3A/B and V7) and two lateral higher-tier areas (LO1 and LO2) are shown in Figure 5. Robust responses to the test stimuli in both orthogonal and parallel trials were found in most visual areas, although the responses in V7 and LO2 were weak and variable. The response amplitudes for all visual areas are summarized in Figure $6 \mathrm{~A}$. Responses in early visual areas were generally larger than those in higher-tier visual areas; mean response amplitudes were $\sim 0.6 \%$ in $\mathrm{V} 1$ and $\sim 0.5 \%$ in $\mathrm{V} 2$ and $\mathrm{V} 3$, whereas the response amplitudes were smaller $(\sim 0.3 \%)$ in ventral higher-tier areas (hV4 and VO1) and smaller still $(\sim 0.1 \%)$ in dorsal and lateral higher-tier visual areas (V7 and LO2).

\section{Adaptation increases from early to higher-tier visual areas}

To quantify the amount of adaptation relative to the overall response to the test stimuli, we calculated an adaptation index (see 

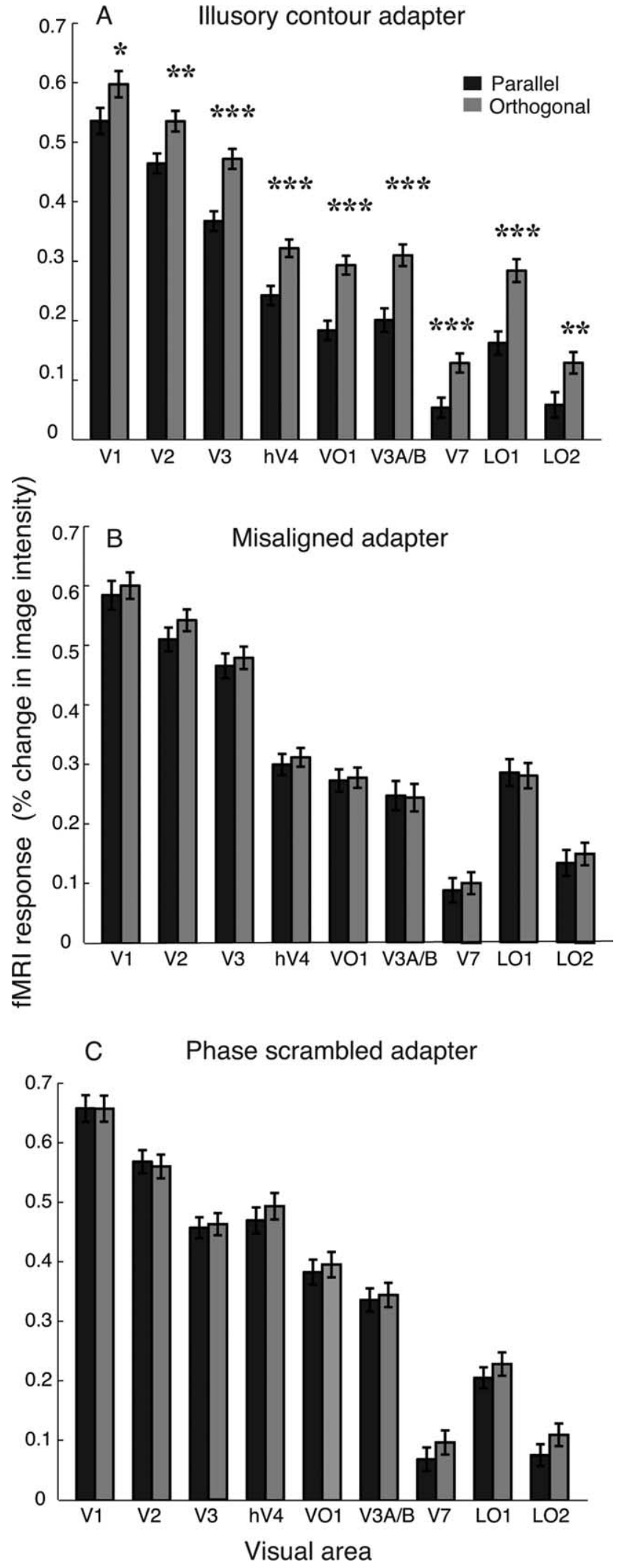

Figure 6. Response amplitudes, averaged across observers, for all visual areas. $\boldsymbol{A}$, Main experiment (illusory contour adapter). $\boldsymbol{B}$, First control experiment (misaligned adapter). $\boldsymbol{C}$, Second control experiment (phase-scrambled adapter). Light bars, Response amplitudes for orthogonal test stimuli. Dark bars, Response amplitudes for parallel test stimuli. Asterisks indicate a statistically significant difference between group average responses to orthogonal and parallel tests $\left({ }^{*} p<0.05 ;{ }^{* *} p<0.01 ;{ }^{* * *} p<0.001\right.$ ) (Table 1). Error bars represent SEM across four observers.
Materials and Methods). Adaptation indices in all visual areas, averaged across observers, are plotted in Figure 7A. Adaptation indices were significantly different from zero showing orientation-selective adaptation to illusory contours in all visual areas studied.

Although we measured larger absolute responses to illusorycontour stimuli in early visual areas, the adaptation indices were larger in higher-tier visual areas. Specifically, the adaptation index was small $(\sim 0.1)$ in V1 and significantly larger $(\sim 0.4)$ in higher-tier areas such as $\mathrm{V} 7$ and $\mathrm{LO} 2$ (Fig. $7 \mathrm{~A}$ ), indicating that adaptation differed across visual areas, and the relative amount of response adaptation increased from early to higher-tier visual areas.

No orientation-selective adaptation to the control stimuli We argue that the adaptation effects in the main experiment are caused by the reduction of responses in neurons that are selective for the orientation of illusory contours. However, we first prove that these response reductions were not the result of incidental properties of the stimuli unrelated to the illusory contours per se. Two control experiments were designed to rule out two such features of the stimuli: the pattern of the line segments and the distribution of energy across the Fourier spectrum.

A version of our illusory contour stimuli with regular spacing of inducer lines (no jitter) forms a distinct pattern, with diagonal line segments in regular columns (for a vertical illusory-contour stimulus) and regular shifts of line segments from one column to the next. Although the irregular spacing (i.e., jitter) of the inducer lines (see Materials and Methods) (Fig. 1A) served to perceptually mask this inducer line pattern, the underlying pattern could have contributed to the observed adaptation. The first control experiment used misaligned adapter stimuli (Fig. $1 B$ ) to rule out this possibility. The misaligned adapter stimuli contained a similar underlying inducer line pattern as the (non-misaligned) adapter stimuli used in the main experiment but did not contain illusory contours. With the misaligned adapter stimuli, no visual area showed orientation-selective response adaptation to the illusory-contour test stimuli (Fig. 6B, Table 1) and hence no adaptation indices differed significantly from zero (Fig. $7 B$ ). Thus, orientation-selective adaptation in the main experiment is not likely caused by an incidental effect of the pattern of phaseshifted inducers in the illusory-contour stimuli.

The horizontal and vertical illusory-contour stimuli do not have identical Fourier power spectra. The spatial phase of inducers and illusory contours was changed each $160 \mathrm{~ms}$ during the adaptation period, and thus it is unlikely the adaptation effects were caused by neurons with linear receptive fields. Even so, the adaptation effects in the main experiment could have resulted from adaptation of neurons selective for the orientation and spatial frequency content of the stimuli (Ginsburg, 1975; Skottun, 1994). In the second control experiment, the adaptor stimuli were phase-randomized versions of the illusory-contour stimuli (Fig. 1C). Therefore, if the adaptation was attributable to neurons selective for the distribution of spectral power, then orientationselective adaptation should have been obtained again with these phase-randomized adapters. However, no area showed a significant difference in response to the orthogonal versus the parallel test stimuli (Fig. 6C, Table 1), and no adaptation index was significantly different from zero (Fig. 7C).

\section{Psychophysics}

The results of the behavioral experiment confirmed that the adapter stimuli were behaviorally effective in eliciting adaptation 

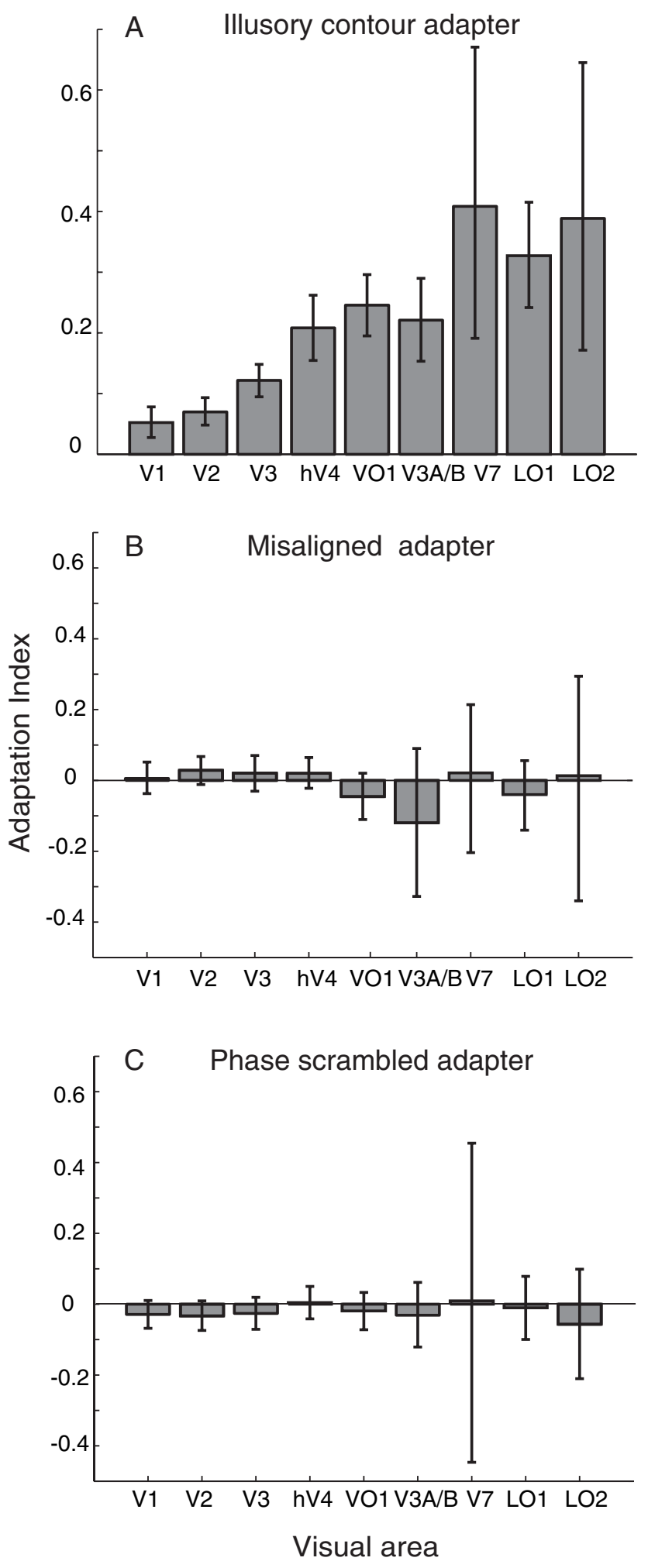

Figure 7. Adaptation indices, averaged across observers, for all visual areas. $A$, Main experiment (illusory contour adapter). Error bars represent $84 \%$ confidence intervals for the mean of the four observers estimated from bootstrap-generated distributions of means in individual observers. Visual areas beyond V2 exhibited significantly larger adaptation indices than area V1 $(p<0.05)$. B, First control experiment (misaligned adapter). $\boldsymbol{C}$, Second control experiment (phase-scrambled adapter). None of the adaptation indices are statistically different from zero in either control experiment.
A
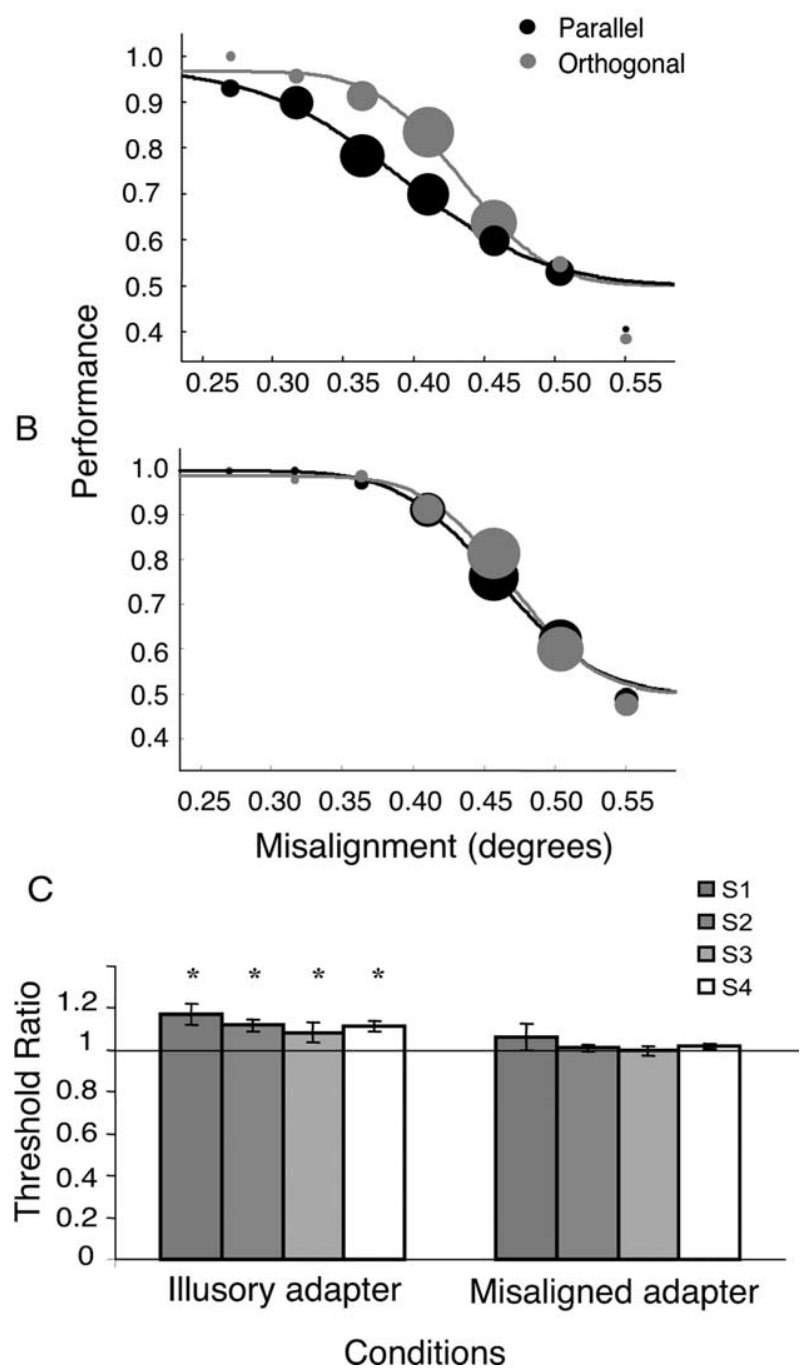

Figure 8. Psychophysical measurements of orientation-selective adaptation. $\boldsymbol{A}$, Example psychometric functions (observer 4) for detecting illusory contours after adaptation to parallel (black) or orthogonal (gray) illusory contours. Performance is plotted as a function of the misalignment of the test stimuli (the adapters were not misaligned). The size of the plot symbols corresponds to the number of trials at each test misalignment level. Smooth curves indicate best-fit (maximum-likelihood) psychometric functions. $\boldsymbol{B}$, Examples of psychometric functions (observer 4) for detecting illusory contours after adaptation to parallel or orthogonal misaligned stimuli. C, Ratios between postadaptation detection thresholds (defined as $75 \%$ correct) measured with test stimuli parallel and orthogonal to illusory-contour adapter stimuli (without misalignment) and to misaligned adapter stimuli. Asterisks indicate statistically significant threshold decrement for parallel test stimuli $\left({ }^{*} p<0.05\right)$. Error bars represent $84 \%$ confidence interval for the threshold ratios, estimated from bootstrap-generated distributions of threshold ratios in each individual observer.

to illusory contours in the main experiment but not in the first control experiment (Fig. 8). For the illusory-contour adapter stimuli, discrimination thresholds were higher for the parallel (adapted) than for the orthogonal (nonadapted) test stimuli, indicating orientation-selective adaptation $(p<0.05)$. In contrast, when the misaligned stimuli were used as adapters, detection thresholds for the parallel and orthogonal test stimuli did not differ significantly ( $p=0.137)$. Note that, unlike the fMRI experiment, attention was not diverted from the illusory-contour stimuli in the behavioral experiment. It is possible that the adaptation in the fMRI data would have been stronger if observers had attended to the illusory contour stimuli (see Discussion). There- 
fore, the degree of orientation-selective adaptation is not directly comparable in the two experiments, but it is reassuring nonetheless to have found a perceptual effect of adaptation using the same stimuli.

\section{Discussion}

Our results indicate that there are neurons selective for illusorycontour orientation in most retinotopically organized human visual areas. The lack of significant adaptation in the control experiments confirms that the adaptation found in the main experiment was in response to illusory contours and not to incidental features of the spatial pattern of line inducers or the distribution of spectral energy. These findings are consistent with singleunit recordings in cats and monkeys that found neurons selective for the orientation of illusory contours in V1 and V2 (Redies et al., 1986; Sheth et al., 1996; Lee and Nguyen, 2001) and also confirm imaging studies showing that illusory contours evoke responses in higher-tier visual areas (Mendola et al., 1999; Murray et al., 2002; Stanley and Rubin, 2003). Our results extend these studies by demonstrating orientation-selective responses in higher-tier visual areas beyond V2.

\section{Origin of extrastriate adaptation}

We found orientation-selective adaptation to illusory contours in multiple visual areas. A decreased response to the adapted stimulus in higher-tier areas could be the result of decreased input resulting from adaptation in earlier areas. Such "inherited adaptation" propagating from earlier areas (Krekelberg et al., 2006) has been observed in single-unit recording studies (Kohn and Movshon, 2004; Tolias et al., 2005). Kohn and Movshon (2004) showed that direction-selective response adaptation in MT neurons was the result of adaptation in direction-selective neurons in V1 that project to MT. In addition, Larsson et al. (2006) suggested that orientation-selective adaptation to luminance-defined gratings found in human extrastriate visual areas such as V2 and V3 could be accounted for by propagation of adaptation from V1.

In our experiment, adaptation indices increased significantly from V1 and V2 to higher-tier visual areas. This result indicates either that there was a greater proportion of neurons that exhibited orientation-selective adaptation in higher-tier areas than in early visual areas, or that the adaptation was stronger in highertier areas for each neuron that adapted. This suggests that response adaptation in these higher-tier visual areas was not trivially inherited from V1. If inherited, it would have to be the case that higher-tier areas received a dominant input from the subpopulation of V1 neurons that exhibited orientation-selective responses to illusory contours. Otherwise, our results imply that there was additional adaptation in higher-tier visual areas.

The progressive increase in orientation-selective adaptation from V1 to higher-tier visual areas might reflect the progressive increase in receptive field sizes across those cortical areas. Given the larger receptive fields in higher-tier visual areas compared with V1 and V2, a larger portion of our stimulus (more illusory contours) fell within the receptive fields of the neurons in highertier visual areas, which might have contributed to the greater adaptation observed in those areas. Furthermore, the adaptation in V1 and V2 might have been stronger had the spatial frequency of the illusory contours been higher than what we used and hence better matched to the spatial frequency tuning of V1 and V2 neurons. That is, the increase in adaptation from V1 to highertier visual areas may not have been caused by differences in illusory contour processing per se. However, it is believed that neurons in successively higher visual areas perform additional processing, meaning that they respond differently, even after compensating for receptive field size differences. For example, it was reported that monkeys with V4 lesion exhibited a significant deficit in recognizing illusory contours (De Weerd et al., 1996), whereas those with IT lesion had an impaired ability to discriminate shapes defined by illusory contours (Huxlin et al., 2000), suggesting that neurons in higher-tier visual areas represent different properties of illusory contours or shapes defined by such contours even after compensating for receptive field size. Therefore, we find it unlikely that the observed difference in orientation-selective adaptation between visual areas is simply because of a fortuitous choice of spatial frequency.

It is also possible that the adaptation seen in early visual areas could have been the result of adaptation in higher-tier visual areas containing neurons selective to illusory contours and that this adaptation propagated back to early visual areas (including V1) via feedback connections. We cannot determine from the current data whether the adaptation observed in early visual areas was a result of independent adaptation in those areas or inherited by feedback from selective adaptation in another upstream extrastriate area.

It is possible that the adaptation in V1 would have been stronger if observers had attended to the illusory contour stimuli (i.e., without the attention-diverting task), because it is known that attention can strongly modulate adaptation to illusory contours (Montaser-Kouhsari and Rajimehr, 2004). However, attentional modulation of activity is usually found to be weaker in V1 than in higher-tier areas. Thus, if attending to the stimuli were to cause an increase in orientation-selective adaptation, one would expect a disproportionately larger increase in higher-tier areas than in V1.

\section{Comparison between V1 and V2}

Our study is the first to use fMRI response adaptation as a tool to characterize orientation-selective responses to illusory contours. Consistent with the prediction from single-unit data (von der Heydt and Peterhans, 1989; Sheth et al., 1996; Lee and Nguyen, 2001), we found orientation-selective adaptation in V2 (i.e., we demonstrated that there are neurons selective for illusorycontour orientation in V2). We also found orientation-selective adaptation to illusory contours in V1, consistent with some studies (Redies et al., 1986; Grosof et al., 1993; Sheth et al., 1996; Lee and Nguyen, 2001; Ramsden et al., 2001) but not with others (Peterhans and von der Heydt, 1989; von der Heydt and Peterhans, 1989). However, in these latter studies, single units were isolated by assessing their responses to luminance-defined contours. Hence, they would not have found neurons selective for the orientation of illusory contours that did not respond to luminance-defined contours of the same orientation and spatial frequency.

In our data, there was no difference in the amount of adaptation observed in V1 and V2. Our interpretation of this result is that neurons in V1 and V2 respond to illusory contours consistent with previous optical imaging and single-unit recording results (Sheth et al., 1996). It could be that neurons in V1 respond selectively to illusory contours because of feedback signals from V2 neurons. This latter suggestion is supported by single-unit data reporting that the population-averaged response to illusory contours in V1 was delayed relative to those in V2 (Lee and Nguyen, 2001).

In imaging studies, the involvement of V1 and V2 in illusorycontour representation is controversial. Mendola et al. (1999) reported activity related to Kanizsa-type illusory-contour stimuli 
both in V1 and V2, but the activation was weak and found only after sensitive across-subject analysis. However, we observed large fMRI responses in V1 and V2 to illusory contours composed of abutting gratings, enabling us to measure orientation-selective adaptation in V1 and V2. Hirsch et al. (1995) reported activation near the cortical region corresponding to the projection of the vertical meridian between V1 and V2 using Kanizsa-type illusory contours, which is consistent with a role of $\mathrm{V} 1$ in illusory-contour representation. V1 also responds to dynamic Kanizsa-type illusory-contour stimuli (Seghier et al., 2000). Maertens and Pollman (2005) found retinotopic illusory-contour responses in V1 in a perceptual-training design while observers were performing curvature discrimination on the illusory boundaries of Kanizsa figures. However, other studies reported no activation evoked by illusory contours in V1 (Ffytche and Zeki, 1996; Kruggel et al., 2001; Murray et al., 2002; Ritzl et al., 2003; Stanley and Rubin, 2003).

It is difficult to compare our results with previous imaging studies. First, there are significant differences between the methodologies used. Previous imaging studies measured the overall fMRI responses to illusory-contour stimuli relative to control stimuli containing no illusory contours. In contrast, our study measured the orientation selectivity of responses. Second, the absence of V1 activity in previous studies could be a result of less-sensitive scanner hardware (lower field strength and lesssensitive receiver coils), different data analysis techniques, or a failure to define V1 retinotopically. Third, many of the previous studies used the Kanizsa-type illusory contours, which may be processed differently from the abutting-line gratings used in our study (Gurnsey et al., 1992, 1996).

\section{Involvement of higher-tier visual areas in illusory contour perception}

Our data showed orientation-selective responses in higher-tier visual areas (V3, V4v, VO1, V3A/B, V7, LO1, LO2), implying that there are neurons in those areas that selectively responded to the orientation of illusory contours. No single-unit study has measured neuronal responses to illusory contours in higher-tier visual areas (beyond V2). However, there are two lesion studies suggesting that higher-tier visual areas are involved in the processing and representation of illusory contours. In one study, monkeys with V4 ablations exhibited significant deficits in recognizing illusory contours and contours defined by texture, but smaller or no deficits for motion-, color-, and luminance-defined contours (De Weerd et al., 1996). In a second study, lesions of monkey IT (which may be homologous to human VO1, LO1, and LO2) were found to impair a monkey's ability to discriminate shapes defined by illusory contours, suggesting that IT plays a role in illusory-figure perception (Huxlin et al., 2000).

Imaging studies have also demonstrated the involvement of higher-tier visual areas in illusory-contour representation. Consistent with our data in which adaptation was greater in highertier visual areas, Mendola et al. (1999) reported stronger activity in response to illusory contours in $\mathrm{V} 3 \mathrm{~A}, \mathrm{~V} 4 \mathrm{v}, \mathrm{V} 7, \mathrm{~V} 8$, and the LOC compared with early visual areas. We also found evidence that neurons selective for the orientation of illusory contours exist in LO1 and LO2, two retinotopic areas in the LOC located between V3d and MT (Larsson and Heeger, 2006; Larsson et al., 2006). Several other imaging studies have also found that illusory contours evoke fMRI activity in the LOC (Murray et al., 2002; Ritzl et al., 2003; Stanley and Rubin, 2003). However, because the LOC is a large region containing multiple different subregions (Grill-Spector et al., 1999; Sawamura et al., 2005), the illusory- contour-related activity observed in these studies could have been in subregions of the LOC other than LO1 and LO2.

\section{References}

Aguirre GK, Zarahn E, D'Esposito M (1998) The variability of human, BOLD hemodynamic responses. NeuroImage 8:360-369.

Bandettini PA, Jesmanowicz A, Wong EC, Hyde JS (1993) Processing strategies for time-course data sets in functional MRI of the human brain. Magn Reson Med 30:161-173.

Brainard DH (1997) The psychophysics toolbox. Spat Vis 10:433-436.

Brefczynski JA, DeYoe EA (1999) A physiological correlate of the "spotlight" of visual attention. Nat Neurosci 2:370-374.

Chaudhuri A (1990) Modulation of the motion aftereffect by selective attention. Nature 344:60-62.

De Weerd P, Desimone R, Ungerleider LG (1996) Cue-dependent deficits in grating orientation discrimination after V4 lesions in macaques. Vis Neurosci 13:529-538.

DeYoe EA, Carman GJ, Bandettini P, Glickman S, Wieser J, Cox R, Miller D, Neitz J (1996) Mapping striate and extrastriate visual areas in human cerebral cortex. Proc Natl Acad Sci USA 93:2382-2386.

Efron B, Tibshirani R (1993) An introduction to the bootstrap. New York: Chapman and Hall.

Engel SA (2005) Adaptation of oriented and unoriented color-selective neurons in human visual areas. Neuron 45:613-623.

Engel SA, Rumelhart DE, Wandell BA, Lee AT, Glover GH, Chichilnisky EJ, Shadlen MN (1994) fMRI of human visual cortex. Nature 369:525.

Engel SA, Glover GH, Wandell BA (1997) Retinotopic organization in human visual cortex and the spatial precision of functional MRI. Cereb Cortex 7:181-192.

Fang F, Murray SO, Kersten D, He S (2005) Orientation-tuned fMRI adaptation in human visual cortex. J Neurophysiol 94:4188-4195.

Ffytche DH, Zeki S (1996) Brain activity related to the perception of illusory contours. NeuroImage 3:104-108.

Gandhi SP, Heeger DJ, Boynton GM (1999) Spatial attention affects brain activity in human primary visual cortex. Proc Natl Acad Sci USA 96:3314-3319.

Gardner JL, Sun P, Waggoner RA, Ueno K, Tanaka K, Cheng K (2005) Contrast adaptation and representation in human early visual cortex. Neuron 47:607-620.

Gilbert CD, Wiesel TN (1990) The influence of contextual stimuli on the orientation selectivity of cells in primary visual cortex of the cat. Vision Res 30:1689-1701.

Ginsburg AP (1975) Is the illusory triangle physical or imaginary? Nature 257:219-220.

Grill-Spector K, Kushnir T, Edelman S, Avidan G, Itzchak Y, Malach R (1999) Differential processing of objects under various viewing conditions in the human lateral occipital complex. Neuron 24:187-203.

Grosof DH, Shapley RM, Hawken MJ (1993) Macaque V1 neurons can signal "illusory" contours. Nature 365:550-552.

Gurnsey R, Humphrey GK, Kapitan P (1992) Parallel discrimination of subjective contours defined by offset gratings. Percept Psychophys 52:263-276.

Gurnsey R, Poirier FJ, Gascon E (1996) There is no evidence that Kanizsatype subjective contours can be detected in parallel. Perception 25:861-874.

Hirsch J, DeLaPaz RL, Relkin NR, Victor J, Kim K, Li T, Borden P, Rubin N, Shapley R (1995) Illusory contours activate specific regions in human visual cortex: evidence from functional magnetic resonance imaging. Proc Natl Acad Sci USA 92:6469-6473.

Huk AC, Ress D, Heeger DJ (2001) Neuronal basis of the motion aftereffect reconsidered. Neuron 32:161-172.

Huxlin KR, Saunders RC, Marchionini D, Pham HA, Merigan WH (2000) Perceptual deficits after lesions of inferotemporal cortex in macaques. Cereb Cortex 10:671-683.

Jenkinson M, Bannister P, Brady M, Smith S (2002) Improved optimization for the robust and accurate linear registration and motion correction of brain images. NeuroImage 17:825-841.

Kanizsa G (1976) Subjective contours. Sci Am 234:48-52.

Kastner S, Pinsk MA, De Weerd P, Desimone R, Ungerleider LG (1999) Increased activity in human visual cortex during directed attention in the absence of visual stimulation. Neuron 22:751-761. 
Kohn A, Movshon JA (2004) Adaptation changes the direction tuning of macaque MT neurons. Nat Neurosci 7:764-772.

Krekelberg B, Boynton GM, van Wezel RJ (2006) Adaptation: from single cells to BOLD signals. Trends Neurosci 29:250-256.

Kruggel F, Herrmann CS, Wiggins CJ, von Cramon DY (2001) Hemodynamic and electroencephalographic responses to illusory figures: recording of the evoked potentials during functional MRI. NeuroImage 14:1327-1336.

Lankheet MJ, Verstraten FA (1995) Attentional modulation of adaptation to two-component transparent motion. Vision Res 35:1401-1412.

Larsson J (2001) Imaging vision: functional mapping of intermediate visual processes in man. Stockholm: Karolinska Institutet.

Larsson J, Heeger DJ (2006) Two retinotopic visual areas in human lateral occipital cortex. J Neurosci 26:13128-13142.

Larsson J, Amunts K, Gulyas B, Malikovic A, Zilles K, Roland PE (1999) Neuronal correlates of real and illusory contour perception: functional anatomy with PET. Eur J Neurosci 11:4024-4036.

Larsson J, Landy MS, Heeger DJ (2006) Orientation-selective adaptation to first- and second-order patterns in human visual cortex. J Neurophysiol 95:862-881.

Lee TS, Nguyen M (2001) Dynamics of subjective contour formation in the early visual cortex. Proc Natl Acad Sci USA 98:1907-1911.

Maertens M, Pollmann S (2005) fMRI reveals a common neural substrate of illusory and real contours in V1 after perceptual learning. J Cogn Neurosci 17:1553-1564.

Mendola JD, Dale AM, Fischl B, Liu AK, Tootell RB (1999) The representation of illusory and real contours in human cortical visual areas revealed by functional magnetic resonance imaging. J Neurosci 19:8560-8572.

Montaser-Kouhsari L, Rajimehr R (2004) Attentional modulation of adaptation to illusory lines. J Vis 4:434-444.

Murray MM, Wylie GR, Higgins BA, Javitt DC, Schroeder CE, Foxe JJ (2002) The spatiotemporal dynamics of illusory contour processing: combined high-density electrical mapping, source analysis, and functional magnetic resonance imaging. J Neurosci 22:5055-5073.

Ogawa S, Lee TM, Kay AR, Tank DW (1990) Brain magnetic resonance imaging with contrast dependent on blood oxygenation. Proc Natl Acad Sci USA 87:9868-9872.

Pelli DG (1997) The VideoToolbox software for visual psychophysics: transforming numbers into movies. Spat Vis 10:437-442.

Peterhans E, von der Heydt R (1989) Mechanisms of contour perception in monkey visual cortex. II. Contours bridging gaps. J Neurosci 9:1749-1763.

Pouget A, Dayan P, Zemel R (2000) Information processing with population codes. Nat Rev Neurosci 1:125-132.

Ramsden BM, Hung CP, Roe AW (2001) Real and illusory contour processing in area V1 of the primate: a cortical balancing act. Cereb Cortex 11:648-665.

Redies C, Crook JM, Creutzfeldt OD (1986) Neuronal responses to borders with and without luminance gradients in cat visual cortex and dorsal lateral geniculate nucleus. Exp Brain Res 61:469-481.

Rees G, Frith CD, Lavie N (1997) Modulating irrelevant motion perception by varying attentional load in an unrelated task. Science 278:1616-1619.

Ritzl A, Marshall JC, Weiss PH, Zafiris O, Shah NJ, Zilles K, Fink GR (2003) Functional anatomy and differential time courses of neural processing for explicit, inferred, and illusory contours. An event-related fMRI study. NeuroImage 19:1567-1577.

Sawamura H, Georgieva S, Vogels R, Vanduffel W, Orban GA (2005) Using functional magnetic resonance imaging to assess adaptation and size invariance of shape processing by humans and monkeys. J Neurosci 25:4294-4306
Seghier M, Dojat M, Delon-Martin C, Rubin C, Warnking J, Segebarth C, Bullier J (2000) Moving illusory contours activate primary visual cortex: an fMRI study. Cereb Cortex 10:663-670.

Sereno MI, Dale AM, Reppas JB, Kwong KK, Belliveau JW, Brady TJ, Rosen BR, Tootell RB (1995) Borders of multiple visual areas in humans revealed by functional magnetic resonance imaging. Science 268:889-893.

Sheth BR, Sharma J, Rao SC, Sur M (1996) Orientation maps of subjective contours in visual cortex. Science 274:2110-2115.

Shmuel A, Yacoub E, Pfeuffer J, Van de Moortele PF, Adriany G, Hu X, Ugurbil K (2002) Sustained negative BOLD, blood flow and oxygen consumption response and its coupling to the positive response in the human brain. Neuron 36:1195-1210.

Shmuel A, Augath M, Oeltermann A, Logothetis NK (2006) Negative functional MRI response correlates with decreases in neuronal activity in monkey visual area V1. Nat Neurosci 9:569-577.

Shulman GL (1992) Attentional modulation of a figural aftereffect. Perception 21:7-19.

Skottun BC (1994) Illusory contours and linear filters. Exp Brain Res 100:360-364.

Smith AM, Lewis BK, Ruttimann UE, Ye FQ, Sinnwell TM, Yang Y, Duyn JH, Frank JA (1999) Investigation of low frequency drift in fMRI signal. NeuroImage 9:526-533.

Somers DC, Dale AM, Seiffert AE, Tootell RB (1999) Functional MRI reveals spatially specific attentional modulation in human primary visual cortex. Proc Natl Acad Sci USA 96:1663-1668.

Soriano M, Spillmann L, Bach M (1996) The abutting grating illusion. Vision Res 36:109-116.

Spivey MJ, Spirn MJ (2000) Selective visual attention modulates the direct tilt aftereffect. Percept Psychophys 62:1525-1533.

Stanley DA, Rubin N (2003) fMRI activation in response to illusory contours and salient regions in the human lateral occipital complex. Neuron 37:323-331.

Suzuki S (2001) Attention-dependent brief adaptation to contour orientation: a high-level aftereffect for convexity? Vision Res 41:3883-3902.

Tolias AS, Keliris GA, Smirnakis SM, Logothetis NK (2005) Neurons in macaque area $\mathrm{V} 4$ acquire directional tuning after adaptation to motion stimuli. Nat Neurosci 8:591-593.

Tootell RB, Hadjikhani N, Hall EK, Marrett S, Vanduffel W, Vaughan JT, Dale AM (1998) The retinotopy of visual spatial attention. Neuron 21:1409-1422.

Vogels R (1990) Population coding of stimulus orientation by striate cortical cells. Biol Cybern 64:25-31.

von der Heydt R, Peterhans E (1989) Mechanisms of contour perception in monkey visual cortex. I. Lines of pattern discontinuity. J Neurosci 9:1731-1748.

Wandell BA, Brewer AA, Dougherty RF (2005) Visual field map clusters in human cortex. Philos Trans R Soc Lond B Biol Sci 360:693-707.

Wetherill GB, Levitt H (1965) Sequential estimation of points on a psychometric function. Br J Math Stat Psychol 18:1-10.

Wichmann FA, Hill NJ (2001a) The psychometric function: I. Fitting, sampling, and goodness of fit. Percept Psychophys 63:1293-1313.

Wichmann FA, Hill NJ (2001b) The psychometric function: II. Bootstrapbased confidence intervals and sampling. Percept Psychophys 63:1314-1329.

Yeh SL, Chen IP, De Valois KK, De Valois RL (1996) Figural aftereffects and spatial attention. J Exp Psychol Hum Percept Perform 22:446-460.

Zarahn E, Aguirre GK, D’Esposito M (1997) Empirical analyses of BOLD fMRI statistics. I. Spatially unsmoothed data collected under nullhypothesis conditions. NeuroImage 5:179-197. 